\title{
Diet-Derived Circulating Antioxidants and Risk of Stroke: A Mendelian Randomization Study
}

\author{
Rujia Miao, ${ }^{1}$ Jing Li, ${ }^{2}$ Changjiang Meng, ${ }^{3,4}$ Yalan Li, ${ }^{3,4}$ Haibo Tang, ${ }^{5}$ Jie Wang $\mathbb{D},{ }^{3,4}$ \\ Peizhi Deng, ${ }^{3,4}$ and Yao Lu (iD) $1,4,6$ \\ ${ }^{1}$ Health Management Center, The Third Xiangya Hospital, Central South University, Changsha, China \\ ${ }^{2}$ Department of Rehabilitation, The Second Xiangya Hospital, Central South University, Changsha, China \\ ${ }^{3}$ Department of Cardiology, The Third Xiangya Hospital, Central South University, Changsha, China \\ ${ }^{4}$ Clinical Research Center, The Third Xiangya Hospital, Central South University, Changsha, China \\ ${ }^{5}$ Department of Metabolic and Bariatric Surgery, The Third Xiangya Hospital, Central South University, Changsha, China \\ ${ }^{6}$ School of Life Course Sciences, King's College London, London, UK
}

Correspondence should be addressed to Yao Lu; luyao0719@163.com

Rujia Miao and Jing Li contributed equally to this work.

Received 27 October 2021; Revised 30 November 2021; Accepted 22 December 2021; Published 17 January 2022

Academic Editor: Ding-Sheng Jiang

Copyright (C) 2022 Rujia Miao et al. This is an open access article distributed under the Creative Commons Attribution License, which permits unrestricted use, distribution, and reproduction in any medium, provided the original work is properly cited.

Background. Oxidative stress is crucial in stroke pathogenesis. Many cohort-based studies suggested that the intake of exogenous antioxidants originated from food may prevent stroke. However, the corresponding randomized controlled trials did not show diet-derived antioxidants have a protective effect on stroke. Objectives. To examine the association of genetically proxied dietderived antioxidants with stroke risk using Mendelian randomization. Methods. We performed a two-sample Mendelian randomization (MR) analysis to evaluate the causal effect of diet-derived antioxidants on stroke risk. For exposure data, we extracted genetic variants as instrumental variables (IVs) that are strongly associated with frequently used diet-derived antioxidants, including vitamin $\mathrm{C}$, vitamin $\mathrm{E}$ ( $\alpha$-tocopherol, $\gamma$-tocopherol), carotene, retinol, zinc, and selenium, from a largescale genome-wide association study (GWAS). We obtained IVs' corresponding effect estimates on the risk of total stroke and ischemic stroke from a GWAS meta-analysis with 40,585 cases and 406,111 controls. Finally, we applied five types of Mendelian randomization analysis to obtain preliminary MR results and performed four three kinds of sensitivity analysis to verify them. Results. According to the primary MR estimations and further sensitivity analyses, we established two robust associations after Bonferroni correction: genetically proxied circulating $\gamma$-tocopherol was causally associated with total stroke [odds ratio $(\mathrm{OR})=0.68,95 \%$ confidence interval $(\mathrm{CI})(0.52-0.88), p=3.78 E-03$ ] and ischemic stroke $[\mathrm{OR}=0.66,95 \% \mathrm{CI}$ (0.51-0.86), $p=2.34 E-03$ ]. There was no evidence to support the causal effect of other diet-derived antioxidants on the risk of total stroke and ischemic stroke. Conclusion. Our study revealed a protective impact of genetic susceptibility to high circulating $\gamma$-tocopherol levels on stroke risk, providing new information on the potential therapeutic targets for primary stroke prevention.

\section{Introduction}

According to World Health Organization, stroke has become the second-leading cause of total death and the third leading cause of disability worldwide. In 2019, there were 12.2 million incident cases and 101 million prevalent cases of stroke globally, where ischemic stroke accounted for $62.4 \%$. Evidence-based prevention strategies by reducing the exposure to stroke risk factors were vital for relieving the burden of public health. Currently, the established top five risk factors for stroke are high systolic blood pressure, body mass index, fasting plasma glucose, ambient particulate matter pollution, and smoking [1]. Notably, in addition to these conventional risk factors, oxidative stress associated with excessive production of reactive oxygen species (ROS) is involved in the pathogenesis of stroke [2]. High 
concentrations of ROS like prooxidant exceeds the counterbalance ability of antioxidants, resulting in cerebrovascular impairment through cellular and vascular mechanism, including endothelial dysfunction, platelet aggregation, and atherosclerosis [3]. It is not surprising that nonselective antioxidants are potentially protective in stroke prevention via scavenging excessive ROS. In fact, it has been reported that endogenous antioxidant substances, such as bilirubin, are causally associated with decreased stroke risk [4]. Many cohort-based studies on stroke prevention support our hypothesis that stroke-susceptible individuals will benefit from regular intake of diet-derived antioxidants [5-7]. Studies suggested zinc and selenium, as antioxidants and antiinflammatory agents, were inversely associated with the incidence of stroke [8-10]. Conversely, many randomized controlled trials (RCTs) and their meta-analyses have failed to demonstrate that supplementary antioxidants reduce the incidence of stroke [11-13]. It is unclear whether the protective effect of antioxidants on stroke incidence in observational studies was attributed to the bias by confounders such as subjects who preferred healthy diets containing multiple antioxidants or were stuck to a healthier lifestyle. These confounding factors potentially affected cerebrovascular condition. Moreover, reverse causation between dietderived antioxidants and stroke should also be considered.

To overcome the bias from previous studies, we improve the research design by applying Mendelian randomization (MR) analysis that exploited genetic variants as instrumental variables to establish a strong causal inference between exposure levels of common diet-derived antioxidants and risk of total and ischemic stroke without involving confounders and reverse causations [14].

\section{Method}

2.1. Study Design. The general design of the current study is illustrated in Figure 1. First of all, we obtained available genetic variants from the large-scale GWASs for vitamin C (ascorbate), vitamin E ( $\alpha$-tocopherol), vitamin E ( $\gamma$-tocopherol), carotene, vitamin A (retinol), zinc, and selenium. Secondly, we selected the summary data of stroke and ischemic stroke from a GWAS meta-analysis. Finally, the causal relationships between diet-derived antioxidants and stroke risk were assessed by a two-sample MR analysis and several sensitivity analyses.

2.2. Data for Exposures. Our primary exposures were genetically determined diet-derived antioxidants. In this study, a total of seven diet-derived antioxidants were considered: vitamin C (ascorbate), vitamin E ( $\alpha$-tocopherol), vitamin E $(\gamma$ tocopherol), carotene, vitamin A (retinol), zinc, and selenium. We identified single-nucleotide polymorphisms (SNPs) associated with these diet-derived antioxidants as IVs $\left[p<5 \times 10^{-6}\right.$; linkage disequilibrium (LD); $r^{2}<0.001$, LD distance $>10,000 \mathrm{~kb}$ ] from the large-scale GWASs (sample sizes ranging from 2,085 to 64,979$)[15,16]$. The strength of the correlation between SNP and diet-derived antioxidants was expressed as an $F$-statistic. Overall, $F$ - statistic $>10$ suggested a strong correlation between the IVs and antioxidants.

2.3. Data for Outcomes. We selected total stroke and ischemic stroke as the main outcomes in our study. Summary statistics on the association of exposure-related SNPs with outcomes were abstracted from a large meta-analysis including 17 studies for European participants. The dataset involved 40,585 total stroke cases, 34,217 ischemic stroke cases, and 406,111 controls [17]. To our knowledge, there was no sample overlap between the exposure and outcome GWASs.

\subsection{Statistical Analysis}

2.4.1. Two-Sample Mendelian Randomization Analysis. We used the classical MR model to examine the causal relationships between these diet-derived antioxidants and the stroke risk (Figure S1). The selection of IVs includes the following criteria: (i) the IVs must be closely associated with dietderived antioxidants (in this study, defined as the genetic association $p<5 \times 10^{-6}$ ); (ii) not related to confounders of antioxidants and stroke (we conducted a phenome-wide association test to evaluate the relationship between IVs and potential confounders such as body mass index, blood pressure, plasma lipid levels, hypertension, and smoking using PhenoScanner V2 [18]; and (iii) affect stroke only through these antioxidants. In our MR analysis, we used five different methods [inverse-variance weighted (IVW), weighted median, MR-Egger regression, MR-robust adjusted profile score (MR-RAPS), and MR-Pleiotropy Residual Sum and Outlier (MR-PRESSO)] to test the association between dietary antioxidants and stroke or ischemic stroke. The IVW provides MR estimation by combining each Wald ratio of multiple SNPs, showing the largest statistical power among all MR methods [19]. If more than $50 \%$ of the weight comes from valid genetic variation, the weighted median could provide accurate estimations [20]. The MR-Egger regression mainly detects and explains horizontal pleiotropy [21]. Furthermore, we also used MR-PRESSO to detect potential outliers and adjust pleiotropy by removing outliers if necessary [22]. Since we used a relatively higher significant threshold $\left(p<5 \times 10^{-6}\right)$ to select genetic variants, we further performed the MR-RAPS to obtain MR estimations using potentially weak instruments [23].

2.4.2. Sensitivity $M R$ Analyses. In this study, Cochrane's $Q$ test [24], Egger regression intercepts [25], and MRPRESSO global test were used for sensitivity analysis to further examine heterogeneity and horizontal pleiotropy. Cochrane's $Q$ test was applied to quantify heterogeneity across instrumental variables. MR-Egger intercept tests were used to describe the potential horizontal pleiotropy in the analysis. In addition, we performed a leave-one-out test by sequentially removing each SNP and reestimated the MR results. Based on the above analyses, we took IVW as the primary causal effect estimates and considered the consistency across all MR methods. 


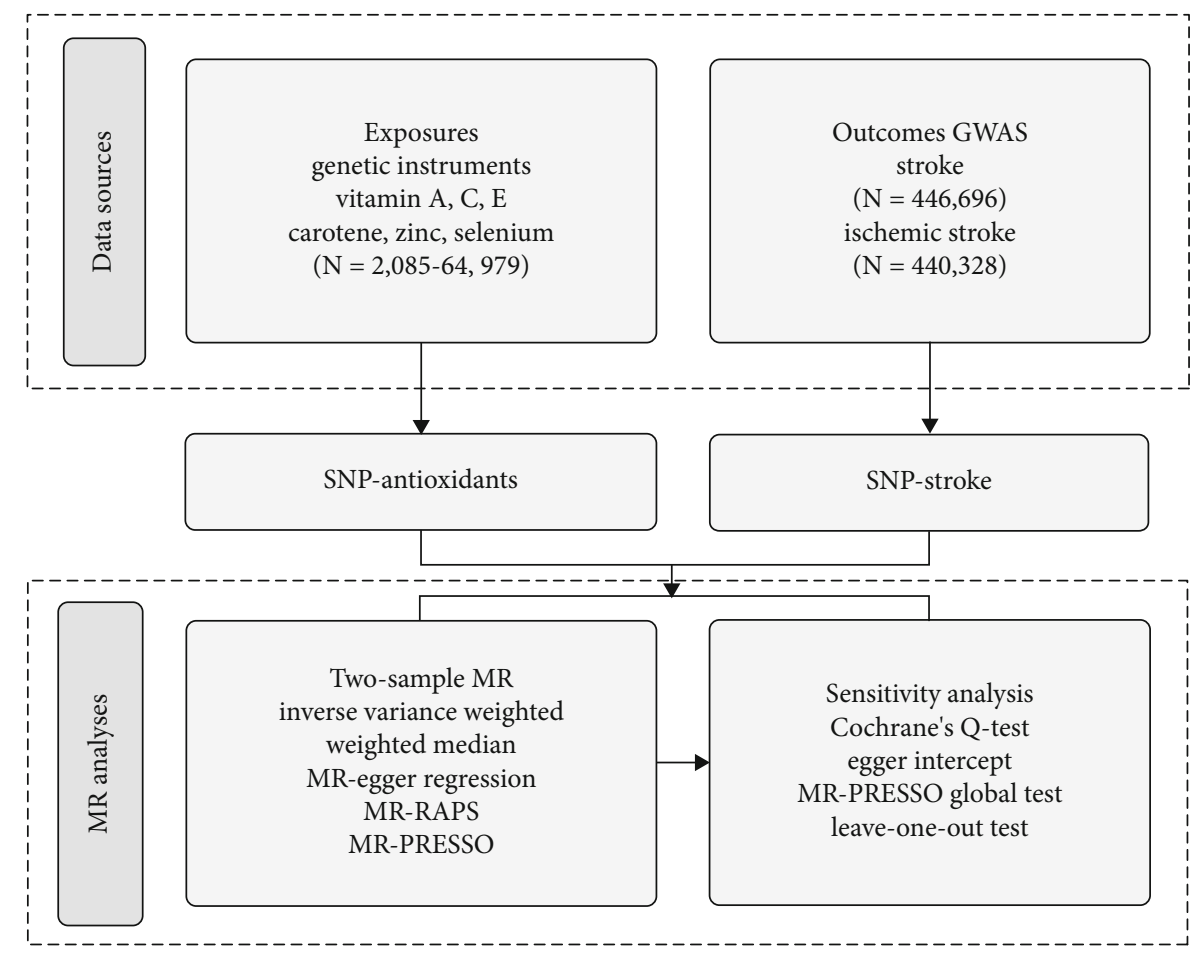

Figure 1: Diagram of Mendelian randomization framework in this study. SNPs for dietary antioxidants (vitamin A, vitamin E, vitamin C, carotene, zinc, and selenium) were identified as genetic instrumental variables. Summary statistics for gene-stroke or gene-ischemic stroke associations were obtained from UK Biobank. For each exposure, MR analyses (primary analysis using inverse-variance weighted (IVW), weighted median, MR-Egger regression, MR-RAPS, MR-PRESSO, and sensitivity analyses using Cochrane's test, Egger intercept, MRPRESSO global test, and leave-one-out test) were performed. GWAS: genome-wide association study; SNP: single-nucleotide polymorphism; MR: Mendelian randomization.

MR analysis was performed in $\mathrm{R}$ (version 4.0.3) with $\mathrm{R}$ packages "TwoSampleMR", "MR-raps", and "MRPRESSO". The multiple comparisons adjusted $p$ value < $0.0007(0.05 / 7)$ after Bonferroni correction was thought to be statistically significant.

\section{Results}

3.1. Exposure and Outcome. The characteristics of the participants from antioxidants and stroke datasets are shown in Table 1. We exhibited SNPs associated with vitamin C (ascorbate), vitamin E ( $\alpha$-tocopherol), vitamin E ( $\gamma$-tocopherol), carotene, vitamin A (retinol), zinc, and selenium in Table S1. Totally, we enrolled 61 SNPs as IVs for seven antioxidants (F-statistics: 20.75-152.28).

3.2. Main Findings. Table 2 and Figure 2 show MR results for the effects of diet-derived antioxidants on stroke and ischemic stroke. There was clear evidence that genetically determined blood $\gamma$-tocopherol level was causally associated with total stroke $[\mathrm{OR}=0.68,95 \% \mathrm{CI}(0.52-0.88), p=3.78 \mathrm{E}$ $-03]$ and ischemic stroke $[\mathrm{OR}=0.66,95 \% \mathrm{CI}(0.51-0.86)$, $p=2.34 E-03]$. The results from other MR methods showed good consistency with IVW $(p<0.05$ in weighted median and MR-RAPS). Although we did not find a potent connection between $\gamma$-tocopherol and total stroke [OR $=0.80,95 \%$ CI (0.21-3.03), $p=7.57 E-01]$ or ischemic stroke $[\mathrm{OR}=0.67,95 \% \mathrm{CI}(0.19-2.45), p=5.75 E-01]$ in MR-
Egger regression, we observed that estimates had similar magnitude and direction with other MR methods. There was little evidence of causal effects for other diet-derived antioxidants on total stroke and ischemic stroke risk.

3.3. Sensitivity Analysis. Cochrane's $Q$ test showed that only in vitamin $C$ (ascorbate) did significant heterogeneity exists with total stroke and ischemic stroke (all $p<0.05$ in IVW and MR-Egger regression). There was no evidence of directional pleiotropy existing for all antioxidants according to MR-Egger intercept and MR-PRESSO global test except for the association between vitamin $\mathrm{C}$ and total stroke $(p<1.00 E-03)$ and ischemic stroke $(p=1.00 E-03)$ (Table 3). Besides, we identified rs68344631 as an outlier SNP $(p<1.00 E-02)$ in the MR-PRESSO outlier test, and then, we found that the outlier-corrected result had a similar range and direction with raw analyses (Table S2). We further performed a phenome-wide association analysis using PhenoScanner V2, suggesting that rs261301 (an IV of $\gamma$ tocopherol) was associated with lipoprotein and cholesterol $(p=4.39 E-24)$. However, we did not find there were any IVs connected with body mass index, blood pressure, smoking, and hypertension (Table S4). Therefore, we recalculated the MR estimation by removing the invalid SNP and found that the association between $\gamma$-tocopherol and total stroke $[\mathrm{OR}=0.68,95 \% \mathrm{CI}(0.50-0.93), p=1.53 \mathrm{E}$ $-02]$ and ischemic stroke $[\mathrm{OR}=0.66,95 \%$ CI (0.49-0.89), $p=6.40 E-03]$ kept consistent with primary analysis 
TABLE 1: Characteristics of diet-derived antioxidants and stroke datasets.

\begin{tabular}{|c|c|c|c|c|c|}
\hline Exposures & Data source & SNP & $F$-statistic & Sample size & Population \\
\hline Vit. C (ascorbate) & Shin et al. & 10 & 23.67 & 2,085 & European \\
\hline Vit. E ( $\alpha$-tocopherol) & Shin et al. & 5 & 71.42 & 7,725 & European \\
\hline Vit. E ( $\gamma$-tocopherol) & Shin et al. & 7 & 55.22 & 6,226 & European \\
\hline Carotene & MRC-IEU & 13 & 76.98 & 64,979 & European \\
\hline Vit. A (retinol) & MRC-IEU & 8 & 136.20 & 62,991 & European \\
\hline Zinc & Evans et al. & 8 & 40.13 & 2,603 & European \\
\hline Selenium & Evans et al. & 6 & 49.70 & 2,603 & European \\
\hline Outcomes & Data source & Studies & Cases/controls & Sample size & Population \\
\hline Stroke & Meta-analysis & 17 & $40,585 / 406,111$ & 446,696 & European \\
\hline Ischemic stroke & Meta-analysis & 17 & $34,217 / 406,111$ & 440,328 & European \\
\hline
\end{tabular}

Vit: vitamin; SNP: single-nucleotide polymorphism.

(Table S3). Finally, in the leave-one-out analyses, we found that the risk estimates of genetically predicted antioxidants in diet and risk of stroke or ischemic stroke kept consistent substantially after excluding one SNP at each time (Figure S2 and Figure S3).

\section{Discussion}

The current MR study demonstrated that genetically proxied higher circulating $\gamma$-tocopherol levels were causally associated with total and ischemic stroke, but we were unable to find significant associations between genetically higher exposures of other typical antioxidants levels (circulating ascorbate, $\alpha$-tocopherol, carotene, retinol, zinc, and selenium) and stroke/ischemic stroke.

An RCT explored the preventive effect of vitamin $\mathrm{E}$ on ischemic stroke when it was used alone, and the outcome remained inconclusive [12]. When exploring the effect of isomer products of vitamin $\mathrm{E}$, a former study found that serum $\gamma$-tocopherol was inversely associated with ischemic stroke in men from a large-scale cohort [26]. In addition to the effect of subtypes, we should notice that genetic predisposition affected the entire life cycle, whereas supplementation only worked during the trial. A low-dose lifelong exposure may accumulate stronger potential biological effects than the temporary high-dose supplements when a long period of time is needed to develop stroke. To our knowledge, this was the first robust causal evidence that $\gamma$ tocopherol could be potentially applied in stroke prevention. With genetic variants as instrumental variables, MR overcame the limitation of considerable confounding factors in observational studies and provided a more precise estimation of causality. A few RCTs suggested that short-term supplementation of a $\gamma$-tocopherol-rich mixture of tocopherols restored vascular endothelial function from hyperglycemia and smoking-induced impairment [27, 28], implying the translational value of $\gamma$-tocopherol in early-stage protection against subsequent atherosclerosis and vascular disease. As to the finding that no effect of $\alpha$-tocopherol was observed, it might result from a paradoxical effect of $\alpha$-tocopherol on oxidation. As the most active form of vitamin $\mathrm{E}$ in human body, previous studies have reported that excessive concentrations of $\alpha$-tocopherol can cause oxidative stress, leading to lipid peroxidation mediated by the tocopherol radicals [29].

The robust null results of circulating ascorbate, carotene, retinol, zinc, and selenium in our study suggested that kinds of long-term exposure to higher levels of antioxidant did not reduce the risk of stroke, which was consistent with early findings from the large-scale RCT and meta-analyses on trials [11, 30-32]. However, there were always exceptions: an RCT [33] found that taking the $\beta$-carotene supplement alone modestly decreased the incidence of cerebral infarction among men with greater alcohol consumption; the case-control trial showed a significant inverse association between plasma retinol and the risk of the first time stroke among Chinese hypertensive adults [34]. These were inconsistent with our results and might be due to the underlying fact that the characteristics of the population included in the MR and RCT did not coincide. Therefore, we assumed that the use of antioxidants was supposed to be selective, at least according to the gender and existing cardiovascular risks, and further population categories should be done. As previously reported similar cases, implementation of MR could be an informative step in the assessment of antioxidant as a chemoprotection targeting cardiovascular diseases: three large databases for genetically predicted antioxidants ( $\alpha$-, $\gamma$-tocopherol, retinol, ascorbate, and carotene) and coronary heart disease (CHD) associations were MR-analyzed; the results did not support the protective effect of these diet-derived antioxidants on CHD risk [35], suggesting the limited CHD prevention benefit from antioxidant supplement. Another MR study indicated that higher circulating vitamin E level might increase the risk of CHD and myocardial infarction [36], thus promoting the reassessment of the safety and efficacy of vitamin E supplementation. Genetically instrumented zinc [37] but not selenium [38] was positively associated with $\mathrm{CHD}$; this implied the underlying risk of microelements with antioxidative capacity in vascular health. For completeness, our outcome revealed the limited value of antioxidants in cerebrovascular protection, except for $\gamma$-tocopherol as a promising supplement. 


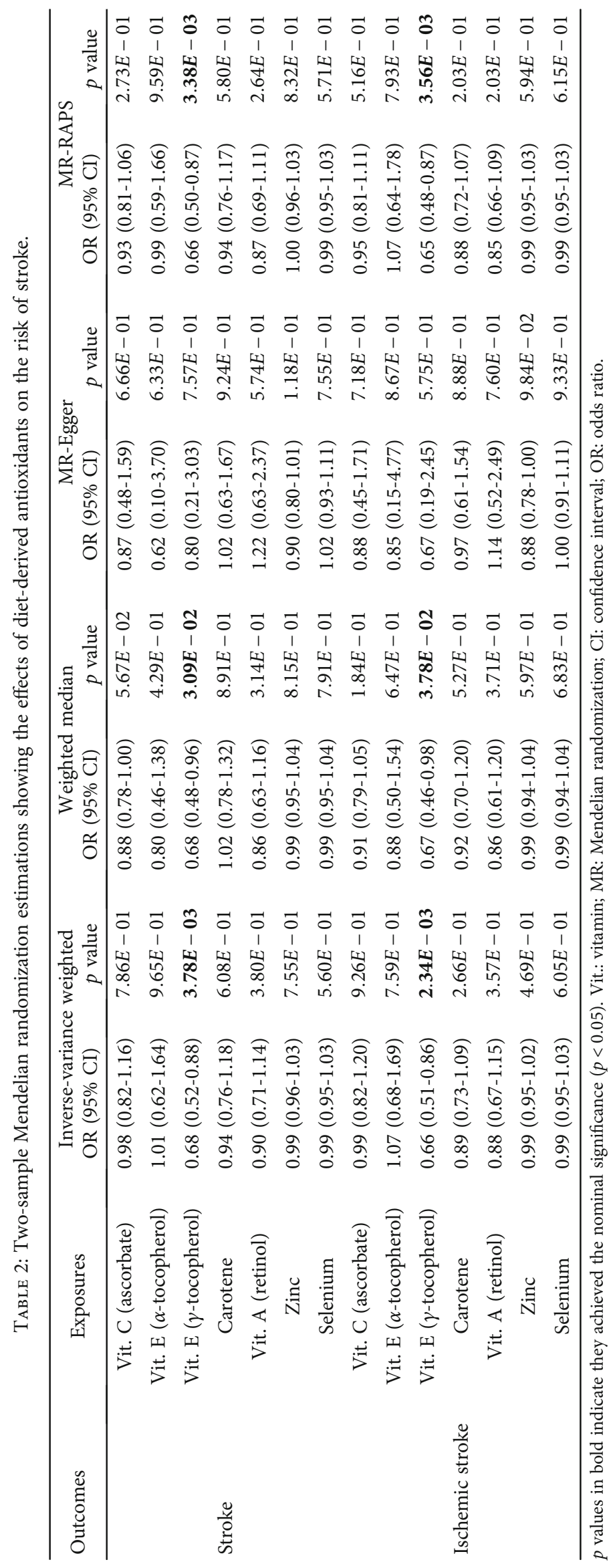




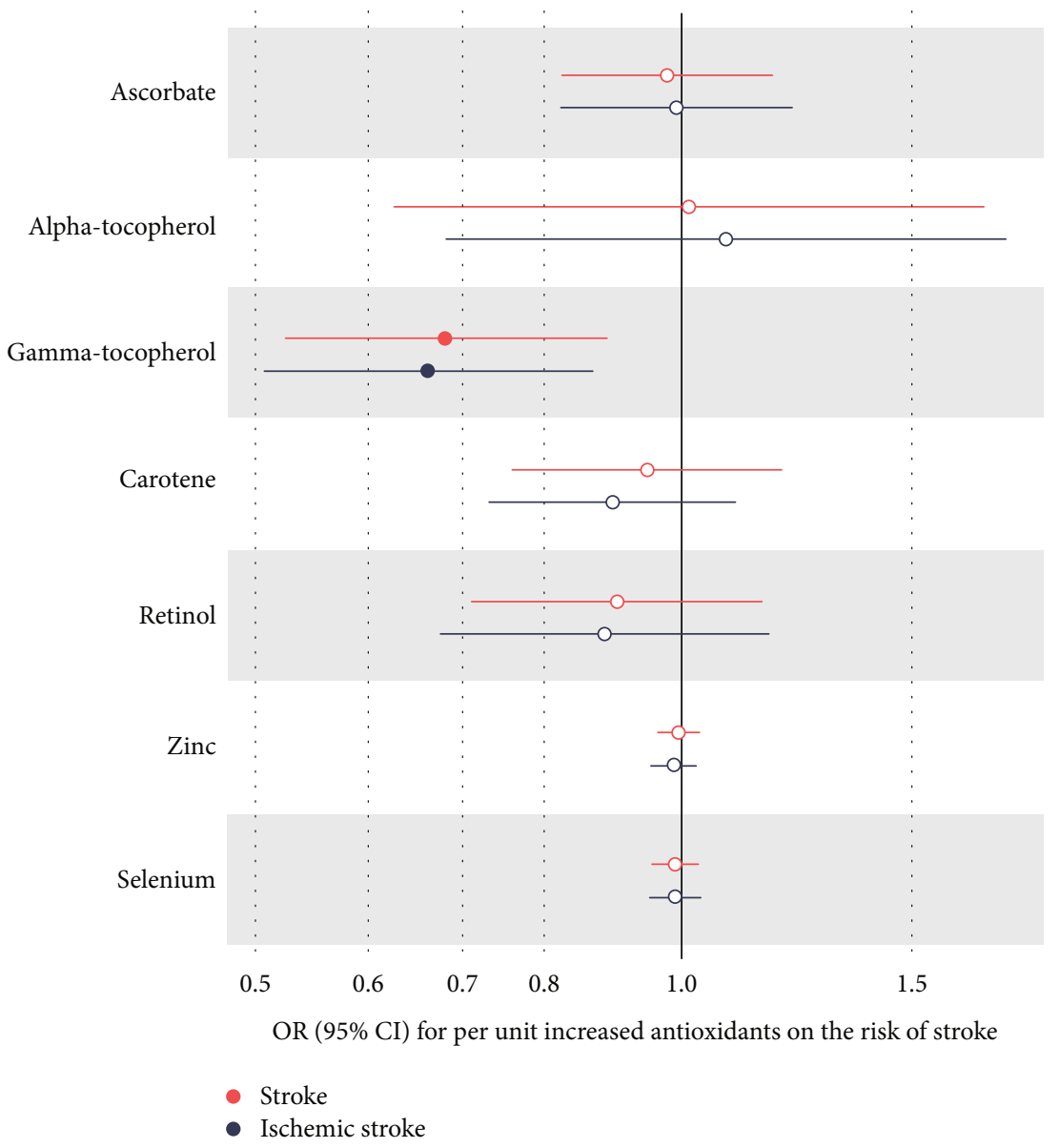

FIgURE 2: The association between genetically determined diet-derived antioxidants and the risk of stroke. Estimated ORs (odds ratio) for the effect of per unit increase in ascorbate, alpha-tocopherol, gamma-tocopherol, carotene, retinol, zinc, and selenium on stroke and ischemic stroke obtained from an inverse-variance weighted (IVW) analysis.

$\gamma$-Tocopherol is the most common form of vitamin $\mathrm{E}$ found in plant seeds and the derivatives [39]. Epidemiological evidence [40] had indicated that intake of $\gamma$-tocopherol but not $\alpha$-tocopherol was significantly inversely associated with the risk of death from cardiovascular disease. Mechanistically, $\gamma$-tocopherol had certain biologically protective properties: $\gamma$-tocopherol was potent in enhancing the activity of superoxide dismutase (SOD) and endothelial cell nitric oxide synthase in arterial tissue, and increasing the expression of both manganese/copper SOD in the antioxidative manner [41, 42]; besides, $\gamma$-tocopherol possessed the capacity of anti-inflammation and antiplatelet aggregation independent of antioxidant activity [41, 43]. Previous studies have reported that $\gamma$-tocopherol may inhibit lipid peroxidation damage and trap reactive nitrogen species [44]. Cooney et al. demonstrated that $\mathrm{NO}_{2}$, also a lipid-soluble species, was sequestered through nitration of $\gamma$-tocopherol which was superior to $\alpha$-tocopherol in the detoxification of $\mathrm{NO}_{2}$ [45]. $\gamma$-Tocopherol may have the potential to scavenge peroxynitrite in inflamed vascular endothelium, thereby limiting the oxidation of $\mathrm{BH} 4$ and helping to preserve effective eNOS activity [46]. With all these potencies, $\gamma$-tocopherol exerted a comprehensive effect against vascular impairment and atherosclerosis originating from diabetes, smoking, aging, and so on.

The strength of the current study was that our MR analysis reflected the impact of lifelong exposure to higher levels of diet-derived antioxidants, considering long-term risks that may not be modified by short-term supplementation treatment. More importantly, MR did not require subjects to be directly exposed to antioxidants, which means it could be implemented at any point without time and resource requirements as RCTs did, thus reducing the possibility of exposing subjects to unnecessary risks and harms [47].

The limitations of our research are as follows: first, the beneficial effects of antioxidants may still exist in unselected subgroups, especially in those with elevated oxidative levels. Besides, traditional treatment plus antioxidants may have a synergistic benefit on stroke. Second, due to the lack of relevant data, we were not able to perform the next step of stroke subtype analysis. Further stroke subtype analysis is needed to determine the correlation between antioxidants and stroke of various subtypes. Third, though no causal association was detected, the potential for the effect size to be too small for identification cannot be fully excluded. Finally, the genetic variants relied on European samples 
TABle 3: The estimations of heterogeneity and horizontal pleiotropy for MR results.

\begin{tabular}{|c|c|c|c|c|c|c|c|c|}
\hline \multirow[b]{2}{*}{ Outcomes } & \multirow[b]{2}{*}{ Exposures } & \multicolumn{2}{|c|}{ IVW } & \multirow[b]{2}{*}{$\begin{array}{c}Q \\
\text {-statistic }\end{array}$} & \multicolumn{2}{|r|}{ MR-Egger } & \multirow[b]{2}{*}{$p$ value } & \multirow{2}{*}{$\begin{array}{l}\text { MR-PRESSO } \\
p \text { for global test }\end{array}$} \\
\hline & & $\begin{array}{c}Q \\
\text {-statistic }\end{array}$ & $p$ value & & $p$ value & Egger intercept & & \\
\hline \multirow{7}{*}{ Stroke } & Vit. C (ascorbate) & 30.83 & $3.16 E-04$ & 30.26 & $1.90 E-04$ & $0.008(-0.033-0.049)$ & $7.07 E-01$ & $<1.00 E-03$ \\
\hline & $\begin{array}{l}\text { Vit. E ( } \alpha- \\
\text { tocopherol) }\end{array}$ & 5.90 & $2.07 E-01$ & 2.05 & $8.43 E-01$ & $0.014(-0.034-0.062)$ & $6.11 E-01$ & $2.69 E-01$ \\
\hline & Vit. E ( $\gamma$-tocopherol) & 6.56 & $3.64 E-01$ & 6.48 & $2.62 E-01$ & $-0.005(-0.042-0.033)$ & $8.16 E-01$ & $3.75 E-01$ \\
\hline & Carotene & 19.24 & $8.30 E-02$ & 19.00 & $6.12 E-02$ & $-0.004(-0.023-0.016)$ & $7.20 E-01$ & $9.20 E-02$ \\
\hline & Vit. A (retinol) & 8.07 & $3.27 E-01$ & 6.97 & $3.24 E-01$ & $-0.012(-0.037-0.012)$ & $3.68 E-01$ & $3.60 E-01$ \\
\hline & Zinc & 5.44 & $6.07 E-01$ & 2.15 & $9.05 E-01$ & $0.022(-0.002-0.045)$ & $1.20 E-01$ & $5.43 E-01$ \\
\hline & Selenium & 2.79 & $7.31 E-01$ & 2.38 & $6.66 E-01$ & $-0.006(-0.024-0.012)$ & $5.56 E-01$ & $7.17 E-01$ \\
\hline \multirow{7}{*}{$\begin{array}{l}\text { Ischemic } \\
\text { stroke }\end{array}$} & Vit. C (ascorbate) & 33.50 & $1.09 E-04$ & 32.95 & $6.29 E-05$ & $0.008(-0.037-0.053)$ & $7.25 E-01$ & $1.00 E-03$ \\
\hline & $\begin{array}{l}\text { Vit. E ( } \alpha- \\
\text { tocopherol) }\end{array}$ & 4.74 & $3.15 E-01$ & 4.62 & $2.02 E-01$ & $0.007(-0.040-0.053)$ & $8.00 E-01$ & $3.27 E-01$ \\
\hline & Vit. E ( $\gamma$-tocopherol) & 5.31 & $5.05 E-01$ & 5.31 & $3.79 E-01$ & $-0.001(-0.037-0.036)$ & $9.78 E-01$ & $5.47 E-01$ \\
\hline & Carotene & 12.80 & $3.06 E-01$ & 12.63 & $2.45 E-01$ & $-0.003(-0.021-0.015)$ & $7.16 E-01$ & $3.36 E-01$ \\
\hline & Vit. A (retinol) & 9.05 & $2.49 E-01$ & 8.40 & $2.10 E-01$ & $-0.010(-0.039-0.019)$ & $5.23 E-01$ & $2.88 E-01$ \\
\hline & Zinc & 5.63 & $5.84 E-01$ & 2.31 & $8.89 E-01$ & $0.024(-0.002-0.049)$ & $1.18 E-01$ & $5.10 E-01$ \\
\hline & Selenium & 2.05 & $8.43 E-01$ & 1.93 & $7.48 E-01$ & $-0.003(-0.022-0.016)$ & $7.52 E-01$ & $8.32 E-01$ \\
\hline
\end{tabular}

Vit: vitamin; MR: Mendelian randomization; IVW: inverse-variance weighted.

too much, and this was owing to the lack of corresponding data from Asian populations.

\section{Conclusion}

In summary, we have posted evidence of a causal relationship between circulating diet-derived antioxidant/metabolites levels and decreased risk of total and ischemic stroke. The study provided information that though most null results limit the application of antioxidants in preventing stroke, $\gamma$-tocopherol is a promising chemoprotection targeting stroke incidence.

\section{Data Availability}

The datasets were derived from sources in the public domain: GWAS (https://gwas.mrcieu.ac.uk) and MR-Base (https://www.mrbase.org/).

\section{Ethical Approval}

This study is a secondary analysis conducted through existing GWAS data. The specific ethics and consent statements reviewed in this study can be accessed in the original publication.

\section{Conflicts of Interest}

The authors declare that they have no conflict of interest and competing interests.

\section{Authors' Contributions}

All authors participated in the field survey and data collection. Rujia Miao, Jing Li, and Changjiang Meng drafted the manuscript. Yalan Li, Haibo Tang, Jie Wang, and Peizhi Deng analyzed the data. Yao Lu and Rujia Miao designed the study. Yao Lu obtained the funding. All authors participated in the field survey and data collection, critically revised the manuscript, and gave final approval to the version submitted for publication. Rujia Miao and Jing Li contributed equally to this work.

\section{Acknowledgments}

This work was supported by the National Natural Science Foundation of China (81600357, 82170437 and 81974054), the Outstanding Young Investigator of Hunan Province (2020JJ2056), the Hunan Youth Talent Project (2019RS2014), and the National Key Research and Development Program (2019YFF0216304 and 2019YFF0216305).

\section{Supplementary Materials}

Figure S1: instrumental variable (IV) assumptions of Mendelian randomization. Figure S2: scatter plot (A, C, E, G, I, $\mathrm{K}, \mathrm{M}$ ) and leave-one-out test ( $\mathrm{B}, \mathrm{D}, \mathrm{F}, \mathrm{H}, \mathrm{J}, \mathrm{L}, \mathrm{N})$ for genetically determined antioxidants and risk of stroke. Figure S3: scatter plot (A, C, E, G, I, K, M) and leave-one-out test (B, D, $\mathrm{F}, \mathrm{H}, \mathrm{J}, \mathrm{L}, \mathrm{N}$ ) for genetically determined antioxidants and risk of ischemic stroke. Table S1: single-nucleotide polymorphisms (SNP) associated with diet-derived antioxidants. Table S2: MR-PRESSO outlier-corrected MR analysis for vitamin C (ascorbate) and risk of stroke and ischemic stroke. 
Table S3: two-sample Mendelian randomization estimations showing the effects of vit. E ( $\gamma$-tocopherol) on the risk of stroke and ischemic stroke by removing outlier rs 261301. Table S4: instrumental variable trait of dietary antioxidants in PhenoScanner V2. (Supplementary Materials)

\section{References}

[1] "Global, regional, and national burden of stroke and its risk factors, 1990-2019: a systematic analysis for the Global Burden of Disease Study 2019," Lancet Neurology, vol. 20, no. 10, pp. 795-820, 2021.

[2] C. Sierra, A. Coca, and E. L. Schiffrin, "Vascular mechanisms in the pathogenesis of stroke," Current Hypertension Reports, vol. 13, no. 3, pp. 200-207, 2011.

[3] C. L. Allen and U. Bayraktutan, "Oxidative stress and its role in the pathogenesis of ischaemic stroke," International Journal of Stroke, vol. 4, no. 6, pp. 461-470, 2009.

[4] Y. Choi, S. J. Lee, W. Spiller et al., "Causal associations between serum bilirubin levels and decreased stroke risk: a two-sample Mendelian randomization study," Arteriosclerosis, Thrombosis, and Vascular Biology, vol. 40, no. 2, pp. 437-445, 2020.

[5] C. R. Gale, C. N. Martyn, P. D. Winter, and C. Cooper, "Vitamin $\mathrm{C}$ and risk of death from stroke and coronary heart disease in cohort of elderly people," BMJ, vol. 310, no. 6994, pp. 1563-1566, 1995.

[6] J. M. Leppälä, J. Virtamo, R. Fogelholm, D. Albanes, and O. P. Heinonen, "Different risk factors for different stroke subtypes," Stroke, vol. 30, no. 12, pp. 2535-2540, 1999.

[7] P. Cheng, L. Wang, S. Ning et al., "Vitamin E intake and risk of stroke: a meta-analysis," The British Journal of Nutrition, vol. 120, no. 10, pp. 1181-1188, 2018.

[8] L. Mattern, C. Chen, L. A. McClure et al., "Serum zinc levels and incidence of ischemic stroke: the reasons for geographic and racial differences in stroke study," Stroke, vol. 52, no. 12, pp. 3953-3960, 2021.

[9] S. J. Fairweather-Tait, Y. Bao, M. R. Broadley et al., "Selenium in human health and disease," Antioxidants \& Redox Signaling, vol. 14, no. 7, pp. 1337-1383, 2011.

[10] B. Hennig, P. Meerarani, M. Toborek, and C. J. McClain, "Antioxidant-like properties of zinc in activated endothelial cells," Journal of the American College of Nutrition, vol. 18, no. 2, pp. 152-158, 1999.

[11] Heart Protection Study Collaborative Group, "MRC/BHF Heart Protection Study of antioxidant vitamin supplementation in 20536 high-risk individuals: a randomised placebo-controlled trial," Lancet, vol. 360, no. 9326, pp. 23-33, 2002.

[12] H. C. Loh, R. Lim, K. W. Lee et al., "Effects of vitamin E on stroke: a systematic review with meta-analysis and trial sequential analysis," Stroke and Vascular Neurology, vol. 6, no. 1, pp. 109-120, 2021.

[13] I. M. Lee, N. R. Cook, J. E. Manson, J. E. Buring, and C. H. Hennekens, "Beta-carotene supplementation and incidence of cancer and cardiovascular disease: the Women's Health Study," Journal of the National Cancer Institute, vol. 91, no. 24, pp. 2102-2106, 1999.

[14] M. Bochud and V. Rousson, "Usefulness of Mendelian randomization in observational epidemiology," International Journal of Environmental Research and Public Health, vol. 7, no. 3, pp. 711-728, 2010.
[15] S. Y. Shin, E. B. Fauman, A. K. Petersen et al., "An atlas of genetic influences on human blood metabolites," Nature Genetics, vol. 46, no. 6, pp. 543-550, 2014.

[16] D. M. Evans, G. Zhu, V. Dy et al., "Genome-wide association study identifies loci affecting blood copper, selenium and zinc," Human Molecular Genetics, vol. 22, no. 19, pp. 39984006, 2013.

[17] R. Malik, G. Chauhan, M. Traylor et al., "Multiancestry genome-wide association study of 520,000 subjects identifies 32 loci associated with stroke and stroke subtypes," Nature Genetics, vol. 50, no. 4, pp. 524-537, 2018.

[18] M. A. Kamat, J. A. Blackshaw, R. Young et al., "PhenoScanner V2: an expanded tool for searching human genotypephenotype associations," Bioinformatics, vol. 35, no. 22, pp. 4851-4853, 2019.

[19] S. Burgess, J. Bowden, T. Fall, E. Ingelsson, and S. G. Thompson, "Sensitivity analyses for robust causal inference from Mendelian randomization analyses with multiple genetic variants," Epidemiology, vol. 28, no. 1, pp. 30-42, 2017.

[20] J. Bowden, G. Davey Smith, P. C. Haycock, and S. Burgess, "Consistent estimation in Mendelian randomization with some invalid instruments using a weighted median estimator," Genetic Epidemiology, vol. 40, no. 4, pp. 304-314, 2016.

[21] S. Burgess and S. G. Thompson, "Interpreting findings from Mendelian randomization using the MR-Egger method," European Journal of Epidemiology, vol. 32, no. 5, pp. 377389, 2017.

[22] M. Verbanck, C. Y. Chen, B. Neale, and R. Do, "Detection of widespread horizontal pleiotropy in causal relationships inferred from Mendelian randomization between complex traits and diseases," Nature Genetics, vol. 50, no. 5, pp. 693 698, 2018.

[23] J. Bowden, M. F. Del Greco, C. Minelli, G. Davey Smith, N. A. Sheehan, and J. R. Thompson, "Assessing the suitability of summary data for two-sample Mendelian randomization analyses using MR-Egger regression: the role of the I2 statistic," International Journal of Epidemiology, vol. 45, no. 6, pp. 1961-1974, 2016.

[24] M. F. Greco, C. Minelli, N. A. Sheehan, and J. R. Thompson, "Detecting pleiotropy in Mendelian randomisation studies with summary data and a continuous outcome," Statistics in Medicine, vol. 34, no. 21, pp. 2926-2940, 2015.

[25] J. Bowden, G. Davey Smith, and S. Burgess, "Mendelian randomization with invalid instruments: effect estimation and bias detection through Egger regression," International Journal of Epidemiology, vol. 44, no. 2, pp. 512-525, 2015.

[26] M. Nagao, Y. Moriyama, K. Yamagishi, H. Iso, A. Tamakoshi, and JACC Study Group, "Relation of serum $\alpha$ - and $\gamma$-tocopherol levels to cardiovascular disease-related mortality among Japanese men and women," Journal of Epidemiology, vol. 22, no. 5, pp. 402-410, 2012.

[27] E. Mah, R. Pei, Y. Guo et al., " $\gamma$-Tocopherol-rich supplementation additively improves vascular endothelial function during smoking cessation," Free Radical Biology \& Medicine, vol. 65, pp. 1291-1299, 2013.

[28] E. Mah, S. K. Noh, K. D. Ballard, H. J. Park, J. S. Volek, and R. S. Bruno, "Supplementation of a $\gamma$-tocopherol-rich mixture of tocopherols in healthy men protects against vascular endothelial dysfunction induced by postprandial hyperglycemia," The Journal of Nutritional Biochemistry, vol. 24, no. 1, pp. 196-203, 2013. 
[29] J. M. Upston, A. C. Terentis, and R. Stocker, “Tocopherolmediated peroxidation of lipoproteins: implications for vitamin $\mathrm{E}$ as a potential antiatherogenic supplement," The FASEB Journal, vol. 13, no. 9, pp. 977-994, 1999.

[30] H. D. Sesso, J. E. Buring, W. G. Christen et al., "Vitamins E and $\mathrm{C}$ in the prevention of cardiovascular disease in men: the Physicians' Health Study II randomized controlled trial," Journal of the American Medical Association, vol. 300, no. 18, pp. 2123-2133, 2008.

[31] J. Zhang, J. Cao, Y. Zhang et al., "Baseline plasma zinc and risk of first stroke in hypertensive patients: a nested case-control study," Stroke, vol. 50, no. 11, pp. 3255-3258, 2019.

[32] S. Stranges, J. R. Marshall, M. Trevisan et al., "Effects of selenium supplementation on cardiovascular disease incidence and mortality: secondary analyses in a randomized clinical trial," American Journal of Epidemiology, vol. 163, no. 8, pp. 694-699, 2006.

[33] J. M. Leppälä, J. Virtamo, R. Fogelholm, D. Albanes, P. R. Taylor, and O. P. Heinonen, "Vitamin E and beta carotene supplementation in high risk for stroke: a subgroup analysis of the Alpha-Tocopherol, Beta-Carotene Cancer Prevention Study," Archives of Neurology, vol. 57, no. 10, p. 1503, 2000.

[34] Y. Yu, H. Zhang, Y. Song et al., "Plasma retinol and the risk of first stroke in hypertensive adults: a nested case-control study," The American Journal of Clinical Nutrition, vol. 109, no. 2, pp. 449-456, 2019.

[35] J. Luo, S. le Cessie, D. van Heemst, and R. Noordam, "Dietderived circulating antioxidants and risk of coronary heart disease: a Mendelian randomization study," Journal of the American College of Cardiology, vol. 77, no. 1, pp. 45-54, 2021.

[36] T. Wang and L. Xu, "Circulating vitamin E levels and risk of coronary artery disease and myocardial infarction: a Mendelian randomization study," Nutrients, vol. 11, no. 9, p. 2153, 2019.

[37] H. P. Kodali, B. T. Pavilonis, and C. M. Schooling, "Effects of copper and zinc on ischemic heart disease and myocardial infarction: a Mendelian randomization study," The American Journal of Clinical Nutrition, vol. 108, no. 2, pp. 237-242, 2018.

[38] A. A. Rath, H. S. Lam, and C. M. Schooling, "Effects of selenium on coronary artery disease, type 2 diabetes and their risk factors: a Mendelian randomization study," European Journal of Clinical Nutrition, vol. 75, no. 11, pp. 1668-1678, 2021.

[39] K. H. Wagner, A. Kamal-Eldin, and I. Elmadfa, "Gammatocopherol-an underestimated vitamin?," Annals of Nutrition \& Metabolism, vol. 48, no. 3, pp. 169-188, 2004.

[40] L. H. Kushi, A. R. Folsom, R. J. Prineas, P. J. Mink, Y. Wu, and R. M. Bostick, "Dietary antioxidant vitamins and death from coronary heart disease in postmenopausal women," The New England Journal of Medicine, vol. 334, no. 18, pp. 1156-1162, 1996.

[41] M. Liu, A. Wallmon, C. Olsson-Mortlock, R. Wallin, and T. Saldeen, "Mixed tocopherols inhibit platelet aggregation in humans: potential mechanisms," The American Journal of Clinical Nutrition, vol. 77, no. 3, pp. 700-706, 2003.

[42] T. Saldeen, D. Li, and J. L. Mehta, "Differential effects of $\alpha \backslash$ and $\gamma$-tocopherol on low-density lipoprotein oxidation, superoxide activity, platelet aggregation and arterial thrombogenesis," Journal of the American College of Cardiology, vol. 34, no. 4, pp. 1208-1215, 1999.

[43] Q. Jiang, I. Elson-Schwab, C. Courtemanche, and B. N. Ames, "Gamma-tocopherol and its major metabolite, in contrast to alpha-tocopherol, inhibit cyclooxygenase activity in macrophages and epithelial cells," Proceedings of the National Academy of Sciences of the United States of America, vol. 97, no. 21, pp. 11494-11499, 2000.

[44] A. Cherubini, C. Ruggiero, C. Morand et al., "Dietary antioxidants as potential pharmacological agents for ischemic stroke," Current Medicinal Chemistry, vol. 15, no. 12, pp. 1236-1248, 2008.

[45] R. V. Cooney, A. A. Franke, P. J. Harwood, V. Hatch-Pigott, L. J. Custer, and L. J. Mordan, "Gamma-tocopherol detoxification of nitrogen dioxide: superiority to alpha-tocopherol," Proceedings of the National Academy of Sciences of the United States of America, vol. 90, no. 5, pp. 1771-1775, 1993.

[46] M. F. McCarty, “Gamma-tocopherol may promote effective no synthase function by protecting tetrahydrobiopterin from peroxynitrite," Medical Hypotheses, vol. 69, no. 6, pp. 1367-1370, 2007.

[47] V. M. Walker, G. Davey Smith, N. M. Davies, and R. M. Martin, "Mendelian randomization: a novel approach for the prediction of adverse drug events and drug repurposing opportunities," International Journal of Epidemiology, vol. 46, no. 6, pp. 2078-2089, 2017. 